\title{
Quantitative analysis of metacarpophalangeal joints during active flexion using four-dimensional computed tomography
}

\author{
Kazunori Ishii $^{\text {a }}$, Satoshi Oki ${ }^{\text {b,c, }}{ }^{*}$ Takuji Iwamoto ${ }^{a}$, Masahiro Jinzaki ${ }^{\mathrm{d}}$, Takeo Nagura ${ }^{\mathrm{b}}$, \\ Morio Matsumoto $^{a}$, Masaya Nakamura ${ }^{a}$ \\ ${ }^{a}$ Department of Orthopaedic Surgery, Keio University School of Medicine, Tokyo, Japan \\ ${ }^{\mathrm{b}}$ Department of Biomechanics, Keio University School of Medicine, Tokyo, Japan \\ ${ }^{\mathrm{c}}$ Department of Orthopedics, Saiseikai Utsunomiya Hospital, Tochigi, Japan \\ ${ }^{\mathrm{d}}$ Department of Radiology, Keio University School of Medicine, Tokyo, Japan
}

\section{A R T I C L E I N F O}

\section{Keywords:}

Metacarpophalangeal joint

Finger kinematics

Rotation axis

4DCT

\begin{abstract}
A B S T R A C T
Background: The metacarpophalangeal joint has a unique morphology with a high degree of freedom. However, few studies have analyzed the kinematics of fingers owing to the rapid movement of the small bones involved. The in-vivo kinematics of metacarpophalangeal joints were analyzed by four-dimensional computed tomography (4DCT) and associated with its morphology.

Methods: The flexion motion of the fingers of bilateral hands in 10 volunteers were examined using 4DCT. Iterative surfaces were registered to trace the surface of the proximal phalanges with respect to metacarpals. Rotation angles were calculated using Euler/Cardan angles.

Findings: In the index finger, the proximal phalange supinated to a maximum flexion of $40^{\circ}$ and then pronated, and its range of rotation was larger than the previous reports. In the other fingers, the proximal phalanges continued to supinate during flexion. The helical axis of the proximal phalange passed a point extremely close to the center point of bilateral condyles, and it moved toward the proximal and palmar directions until the middle stage of flexion and toward the proximal and dorsal directions during the late stage of flexion. The translation of the rotation axis was larger in the ring and little fingers.

Interpretation: The rotation in the index finger was larger than previously reported. The helical axes moved in the dorsal direction and proximally during the latter phase of the flexion. These results can be employed to better understand the causes of implant failure of the metacarpophalangeal joints.
\end{abstract}

\section{Introduction}

Each finger (index, middle, ring, and little fingers) has its own kinematics. In general, phalangeal joints are hinge joints that flex and extend the fingers. When grasping and releasing a subject, these fingers converge to scaphoid tuberosity; further, the direction of flexion varies with the motion of the fingers. Among phalangeal joints, metacarpophalangeal (MCP) joints contribute to this variation in direction compared with the distal interphalangeal (DIP) and the proximal interphalangeal (PIP) joints, because the MCP joint is a spherical condylar joint with a high degree of freedom. The metacarpal head has a unique morphology with an asymmetric articular surface and its collateral ligaments are eccentrically attached. Despite these complexities, most of the implants used for MCP joint arthroplasty are hinge- shape. The survival rate of these hinge-type implants is much lower than that of implants in other joints (Goldfarb and Stern, 2003). Differences in kinematics between the intact joint and implant can cause stress in the implant, leading to fracture. We hypothesized that the MCP joint motions include rotation and translation.

A great majority of previous studies have analyzed finger motion using cadaveric hands (Hess et al., 2013; Kerkhof et al., 2016), the motion capture of markers tracked by camera (Buczek et al., 2011; Coupier et al., 2016; Degeorges et al., 2005), and image analyses (Pagowski and Piekarski, 1977; Tamai et al., 1988). However, these studies did not directly evaluate in-vivo kinematics of the bone segments. In cadaver studies, simulators cannot completely simulate finger motion, because many intrinsic muscles, such as interosseous or lumbricalis muscles, coordinate during active flexion and extension.

\footnotetext{
* Corresponding author at: Department of Biomechanics, Keio University School of Medicine, 35 Shinanomachi, Shinjuku-ku, Tokyo 160-8582, Japan.

E-mail address: satoshiohki@gmail.com (S. Oki).
} 
Optoelectronic systems cannot trace finger motions completely, because the bone size is small and finger motions overlap during total grasp motion. Computed tomography (CT) analysis exhibits an advantage in such situations as it can detect all the positions of the bone cortex. A few studies used sequential static CT data to analyze variations in the motion of phalangeal joints (Kataoka et al., 2011). However, the sequence of static images does not reflect active motion accurately.

There have been several reports recently describing joint kinematics using four-dimensional computed tomography (4DCT) (Wang et al., 2018; Zhao et al., 2015) and potential clinical applications (Garcia-Elias et al., 2014; Troupis and Amis, 2013). With sequential CT volume data, the active motion of the bone segments and its relationship with the bone morphology can be directly evaluated.

In this study, we aimed to describe the in-vivo kinematics of the MCP joint in four fingers using 4DCT, and discuss their relationship with the anatomical morphology.

\section{Methods}

\subsection{Participants}

A total of 10 healthy volunteers (five males and five females, with a mean age of 31.7 and range of 26-34 years) without a history of hand trauma or inflammatory diseases were included. None of the subjects have participated in sport activities, such as climbing or competitive contact sports, which are typically associated with degenerative finger disorders. All subjects were confirmed, through computed tomography, to have no degenerative changes in the finger joints of the hand. Nine of the ten volunteers were right-handed. The protocols of the study and expected radiation exposure were explained to all the subjects and their consent to the examination was obtained.

\subsection{DCT examination}

The subjects were instructed to lie down on the CT bed in a prone position; they were then instructed to set their bilateral hands in the CT gantry with their fingers and elbow extended and their shoulders elevated. For $5 \mathrm{~s}$, four fingers (index, middle, ring, and little fingers) were flexed from an extended position to full grip (Fig. 1). All subjects practiced this finger motion before the examination as a rehearsal. All CT examinations were conducted with a $320 \times 0.5 \mathrm{~mm}$ multi-detector CT (Aquilion One, Cannon Medical Systems, Tochigi, Japan). The tube voltage/tube current was set to $80 \mathrm{kV} / 100 \mathrm{~mA}$, and the scan speed was $0.275 \mathrm{~s}$ in the range of $160 \mathrm{~mm}$. The average of the dose length product required for these examinations was approximately $300(\mathrm{mGy}-\mathrm{cm})$. The effective radiation dose was calculated using the conversion factor of $\kappa$ $=0.0002$ for wrists and hands (Biswas et al., 2009); it was explained to the volunteers that the radiation exposure was approximately $0.06 \mathrm{mSv}$. The institutional review board approved this study, and informed consent was obtained from the volunteers.

\subsection{Data analysis}

The data analysis was performed using iterative surface registration with sequential volume CT DICOM data, as in the previous report (Oki et al., 2019).

\subsubsection{Surface reconstruction}

The isolated surface data of the metacarpal and proximal phalanges were constructed. The outer bone cortex of the metacarpal and proximal phalanges was segmented manually from the CT DICOM data in the first frame of the 4DCT, because the frame before the action is almost identical to the static 3DCT. Each bone surface data were reconstructed via image segmentation software (Avizo Lite 9.0.1, FEI Visualization Sciences Group) (Ryan and Walker, 2010). The whole surface data of all 4DCT frames were then created. The entire surface data of each 4DCT frame were automatically reconstructed by setting the threshold for bone cortex as 200 Hounsfield unit. The file format of a 3D surface was processed as standard triangulated language.

\subsubsection{Coordinate system}

The coordinate systems of the metacarpal and proximal phalangeal bones were defined as shown in Fig. 2. The midpoint between the bilateral center points of the condyle was defined as the origin, which was assumed to be the center of the bone head. The $\mathrm{Y}$-axis vector was the principal axis of inertia on the bone cortex. The $\mathrm{X}$-axis vector was the outer product of the $\mathrm{Y}$-axis and the vector passing through the bilateral center points of the condyle, pointing toward the palm direction. The $Z$ -
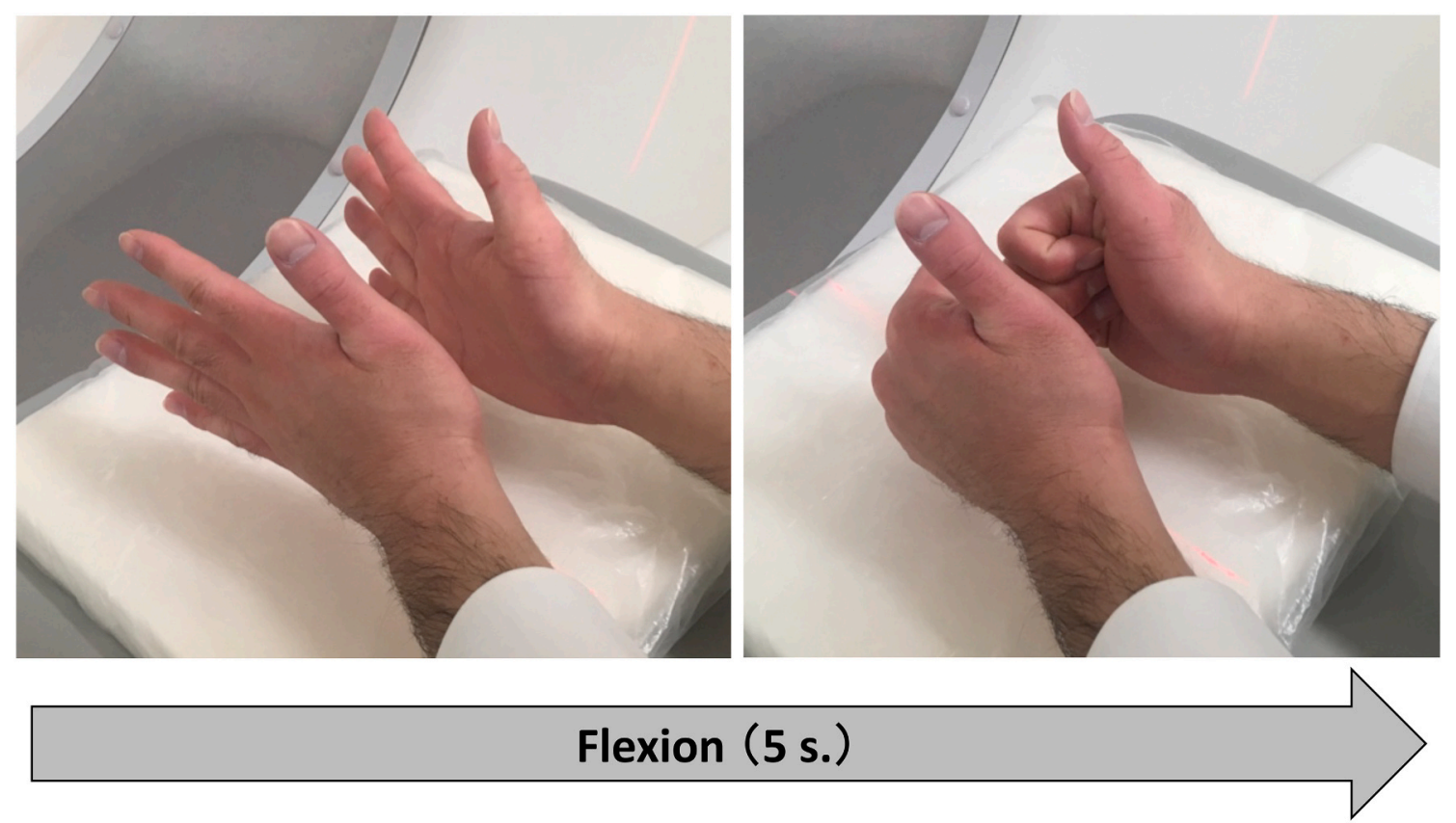

Fig. 1. Volunteers flexed four fingers (they did not flex their thumbs) of their bilateral hands in the CT gantry for $5 \mathrm{~s}$. 


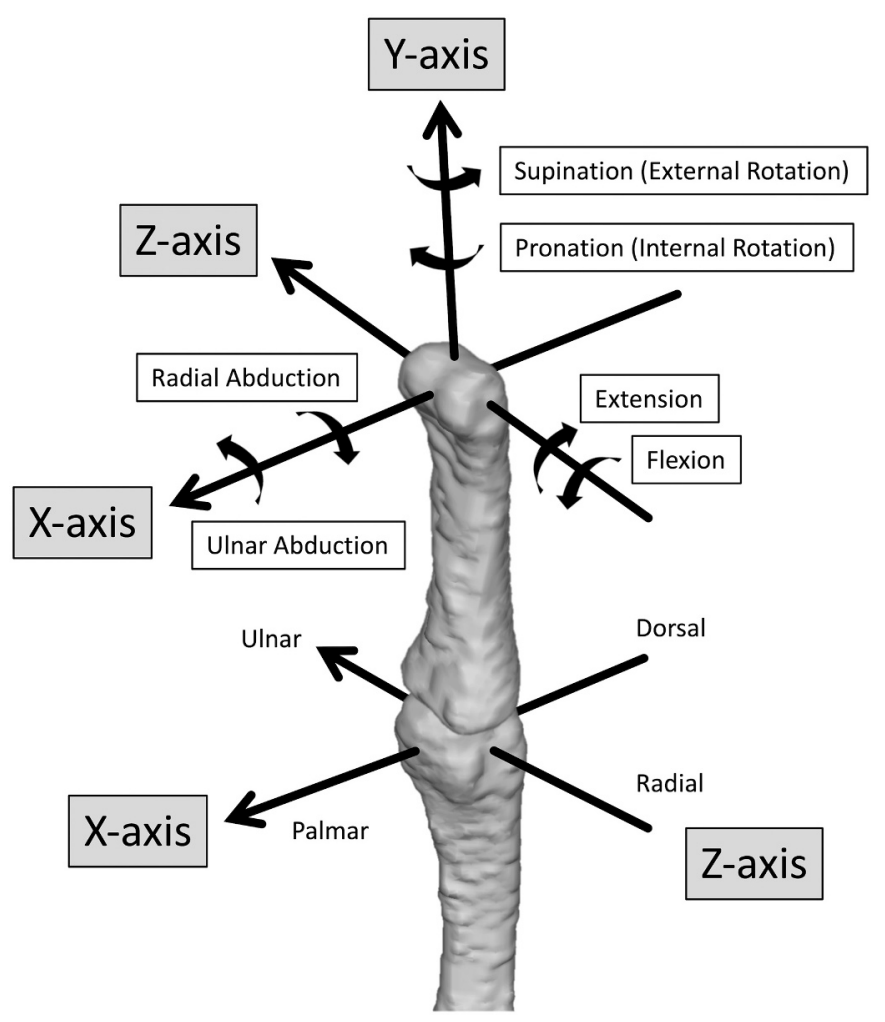

Fig. 2. The coordinate systems of the metacarpal and proximal phalange used the center points of the bilateral condylar region and the vector of the principal axis of inertia on the bone cortex. In addition, the definition of motion was shown. axis was the outer product of the Y- and X-axis (Fig. 2). This definition is similar to the coordinate system that uses the mean helical axis (Coupier et al., 2014).

\subsubsection{Surface registration}

The isolated surface data of the metacarpal and proximal phalanges were registered to $3 \mathrm{D}$ surfaces of the whole hand using an iterative closest point (ICP) algorithm (Fig. 3). The surface data of each segment in the first frame matched with the whole surface data in the next frame since the frame-to-frame motion was sufficiently small for ICP surface registration. The ICP algorithm was repeated iteratively until the last frame of the 4DCT. The accuracy of the surface registration was verified for each frame. When the motion artifact was too large for the ICP algorithm, surface registration was conducted manually by plotting landmarks for each segment.

\subsubsection{Rotation angles}

The $4 \times 4$ matrix required for the surface registration was calculated, and the Euler angles (in order of $\mathrm{z}-\mathrm{x}-\mathrm{y}$ ) of the proximal phalange relative to the metacarpal were calculated. In this coordinate system, the angle rotated around the $\mathrm{X}$-axis was defined as radial-ulnar abduction, around the $\mathrm{Y}$-axis as supination-pronation, and around the $Z$-axis as extensionflexion (Fig. 2). At first, we plotted every 25 frames in the graph. Each parameter was calculated at every $10^{\circ}$ by utilizing the nearest frames for comparison. All other data, except the nearest frames at every $10^{\circ}$, were excluded. Rotation angles at every $10^{\circ}$ of flexion were averaged. In addition, rotation angles at full flexion were also averaged and plotted as the averaged full flexion angles.

\subsubsection{Rotation axis}

The rotation axis was calculated using the helical axis, which is widely used for analyzing kinematics (Kinzel et al., 1972); it describes the rotation axis without parallel displacement. The helical axis was first evaluated in the whole flexion, and then the intersection point of the

\section{Segmented 3D Surface 3D Surface of the Whole Hand}

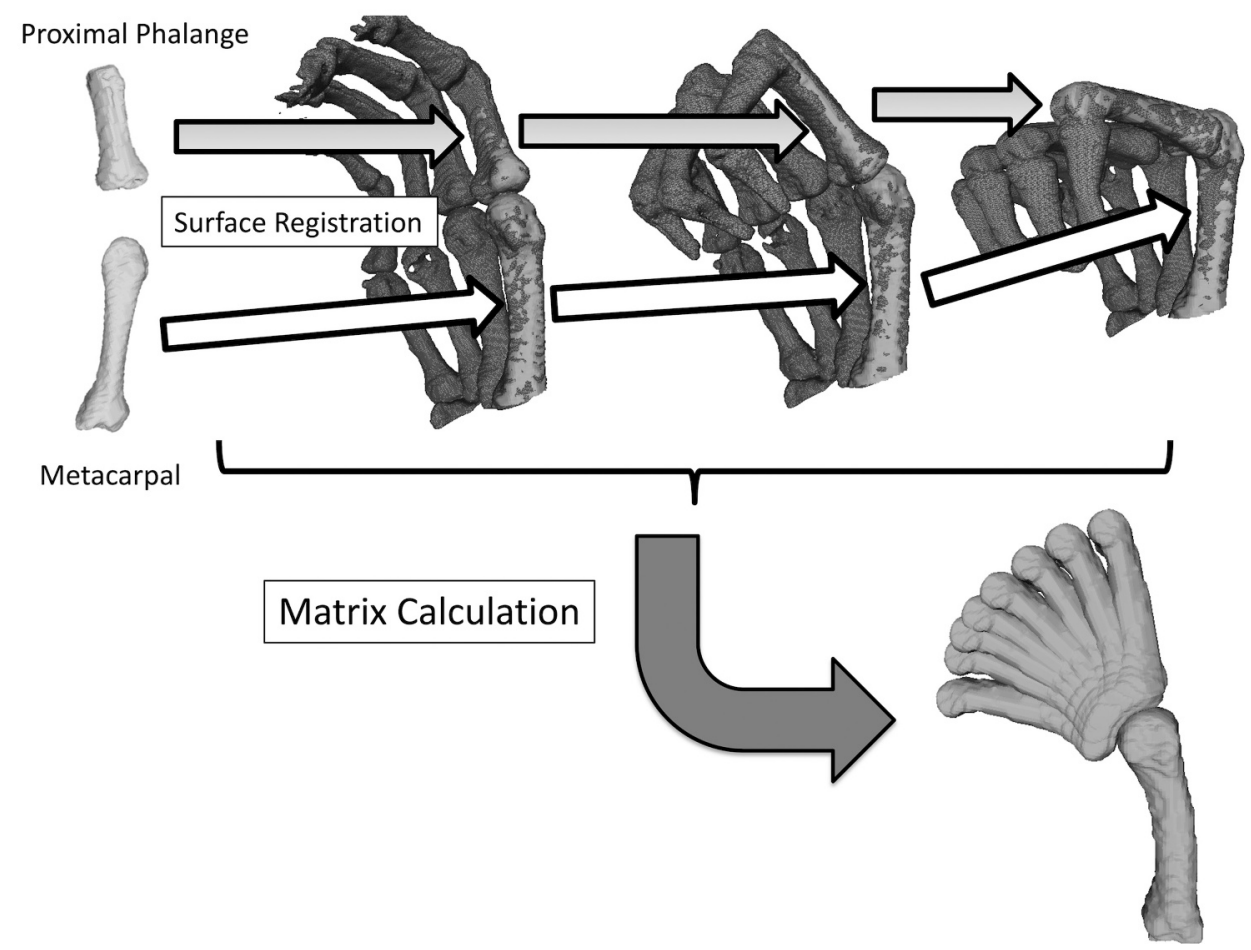

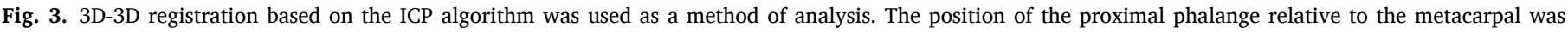
quantified from the calculation of the $4 \times 4$ matrix required for registration. 
helical axis, divided into three flexion stages (up to $30^{\circ}$ of flexion, $30-60^{\circ}$ of flexion, $60^{\circ}$ to full flexion) using the sagittal (X-Y) plane, was measured to investigate the change in the palmar-dorsal and proximaldistal direction of the rotation axis.

\subsubsection{Validation of surface registration}

To validate the accuracy of the surface registration and evaluate how much transformation can be tolerated in the frame-to-frame surface registration, a perturbation analysis was performed. The surface data of the proximal phalange rotated around the $Z$-axis (flexion). The original surface data were matched with the rotated surface data, and the rotation error was calculated. The translation tolerance was also evaluated by translating the surface data along the $\mathrm{X}$-axis (volar translation) and matching it with the original surface as well.

\subsection{Statistics}

The one-way analysis of variance to identify significant differences of the supination-pronation angles or the ulnar-radial adduction angles relative to each flexion phase was conducted using IBM SPSS Statistics (Version24, New York, USA). The Dunnett multiple comparison post hoc test was conducted considering $0^{\circ}$ of flexion. The significance level was set with a $P$ value less than 0.05 .

\section{Results}

\subsection{Rotation angles}

The average range of flexion angles was $-1.5-76.4^{\circ}$ in the index finger, $-3.7-81.8^{\circ}$ in the middle finger, $-3.7-74.9^{\circ}$ in the ring finger, $-8.2-63.5^{\circ}$ in the little finger. The average of the supination-pronation angles (Fig. 4-A) and the radial-ulnar abduction angles (Fig. 4-B) was calculated at every $10^{\circ}$ of flexion and at full flexion. The time phase that was closest to the flexion angle every $10^{\circ}$ was adopted, although not all $10^{\circ}$ of flexion was satisfied by all the data of the 20 hands. The flexion speed (flexion angle per frame) of each finger at $1 \mathrm{~s}$ intervals is presented in Table 1. The rotation speed of the proximal phalange was on average $5^{\circ}$ per frame, even in the fast phase. The perturbation analysis showed that surface registration could trace the surface up to $15^{\circ}$ of rotation and $15 \mathrm{~mm}$ of translation (Supplemental Fig. 1). From these results, the flexion speed was within a range that could be tracked accurately in frame-to-frame surface registration.

In the index finger, the proximal phalange tended to supinate $6.8^{\circ}$ on average until $40^{\circ}$ of flexion from $0^{\circ}(p<0.05)$. After $40^{\circ}$ of flexion, the proximal phalange pronated $2.9^{\circ}$ on average until full flexion, and the pronation-supination angle was no longer significant compared with $0^{\circ}$ of flexion $(p=0.28)$. In the other fingers, the proximal phalanges tended to supinate during flexion $\left(7.2^{\circ}\right.$ in the middle finger $(p<0.05)$, $7.6^{\circ}$ in the ring finger $(p<0.05)$, and $9.7^{\circ}$ in the little finger $(p<0.05)$ ). When the fingers were extended, the abduction angle of the proximal phalange relative to the metacarpal abducted ulnarly in the index finger and radially in the ring and little fingers to converge these fingertips. Each radial or ulnar abduction angle approached $0^{\circ}$ accompanied with flexion. The proximal phalange abducted radially $13.7^{\circ}$ on average in the index finger $(p<0.05)$ during flexion, and it abducted ulnarly in the ring $\left(5.4^{\circ}(p<0.05)\right)$ and little fingers $\left(12.3^{\circ}(p<0.05)\right)$. It was not significantly abducted in the middle finger $(p=0.99)$. No left-right or sex differences were observed. In addition, the pronation-supination and radial-ulnar abduction patterns were not significantly different between the dominant and non-dominant hands (Supplement Fig. 2).

\subsection{Helical axes}

The helical axes of the proximal phalanges during whole flexion on the metacarpal head were close to the center point of bilateral condyles (origin) (Fig. 5). On average, the helical axis moved toward the palmar
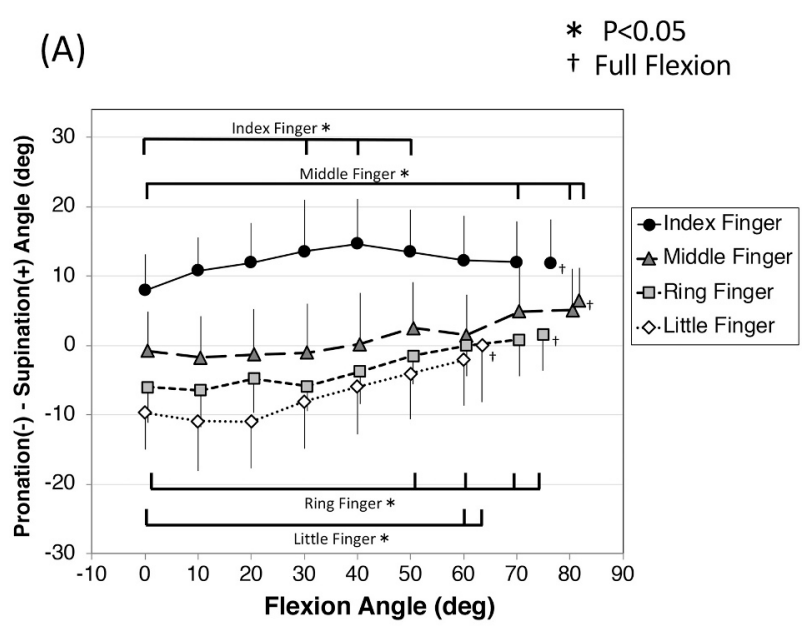

(B)

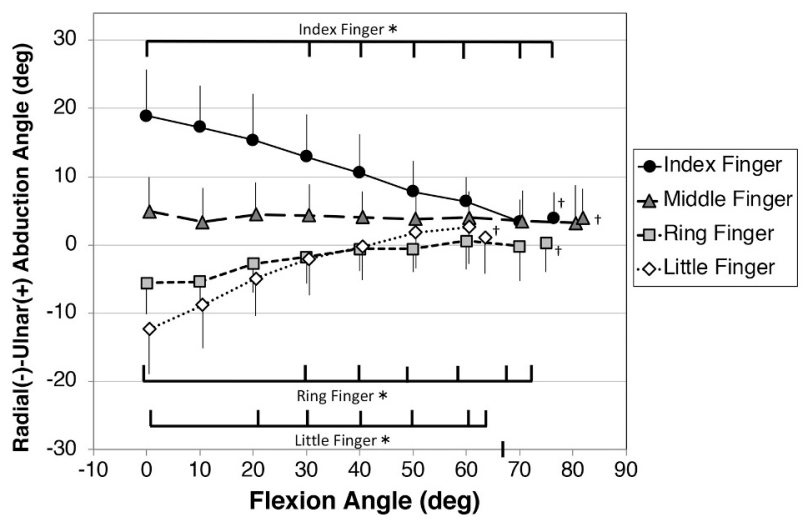

Fig. 4. Average angle (the bars show standard deviation) of pronation(-)-supination $(+)(\mathrm{A})$ and $\operatorname{radial}(-)$-ulnar $(+)$ abduction (B) of the proximal phalange relative to the metacarpal during flexion.

Table 1

The flexion speed of each finger was presented every $1 \mathrm{~s}$ (R: Right hand, L: Left hand). Data are means of flexion degree per frame and (standard deviation). One frame is $0.2 \mathrm{~s}$.

\begin{tabular}{llllll}
\hline Finger & $0-1 \mathrm{~s}$ & $1-2 \mathrm{~s}$ & $2-3 \mathrm{~s}$ & $3-4 \mathrm{~s}$ & $4-5 \mathrm{~s}$ \\
\hline Index finger & R: 1.3 & R: 3.7 & R: 4.5 & R: 4.1 & R: 2.3 \\
& $(1.3)$ & $(2.4)$ & $(2.0)$ & $(1.3)$ & $(1.3)$ \\
& L: 1.0 & L: 4.0 & L: 4.3 & L: 4.0 & L: 1.8 \\
Middle & $(1.0)$ & $(3.0)$ & $(1.2)$ & $(1.6)$ & $(1.3)$ \\
finger & R: 1.3 & R: 3.8 & R: 4.9 & R: 4.5 & R: 2.6 \\
& $(1.5)$ & $(1.9)$ & $(1.8)$ & $(1.3)$ & $(1.4)$ \\
& L: 1.3 & L: 4.4 & L: 4.5 & L: 4.3 & L: 2.3 \\
Ring finger & $(1.2)$ & $(2.6)$ & $(1.6)$ & $(1.6)$ & $(0.9)$ \\
& R: 1.0 & R: 2.2 & R: 3.9 & R: 5.2 & R: 3.7 \\
& $(1.3)$ & $(1.3)$ & $(1.9)$ & $(1.8)$ & $(1.5)$ \\
& L: 1.1 & L: 3.4 & L: 3.6 & L: 4.3 & L: 2.9 \\
& $(1.4)$ & $(1.7)$ & $(1.8)$ & $(2.3)$ & $(1.3)$ \\
Little finger & R: 0.6 & R: 1.3 & R: 2.9 & R: 5.3 & R: 4.4 \\
& $(0.7)$ & $(1.3)$ & $(1.9)$ & $(2.6)$ & $(2.2)$ \\
& L: 0.6 & L: 1.4 & L: 2.8 & L: 5.4 & L: 3.3 \\
& $(0.7)$ & $(1.9)$ & $(1.9)$ & $(2.8)$ & $(1.3)$ \\
\hline
\end{tabular}

and proximal directions during flexion from 30 to $60^{\circ}$ and moved toward the dorsal and proximal directions during flexion from $60^{\circ}$ to full flexion (Fig. 6). 
(A)
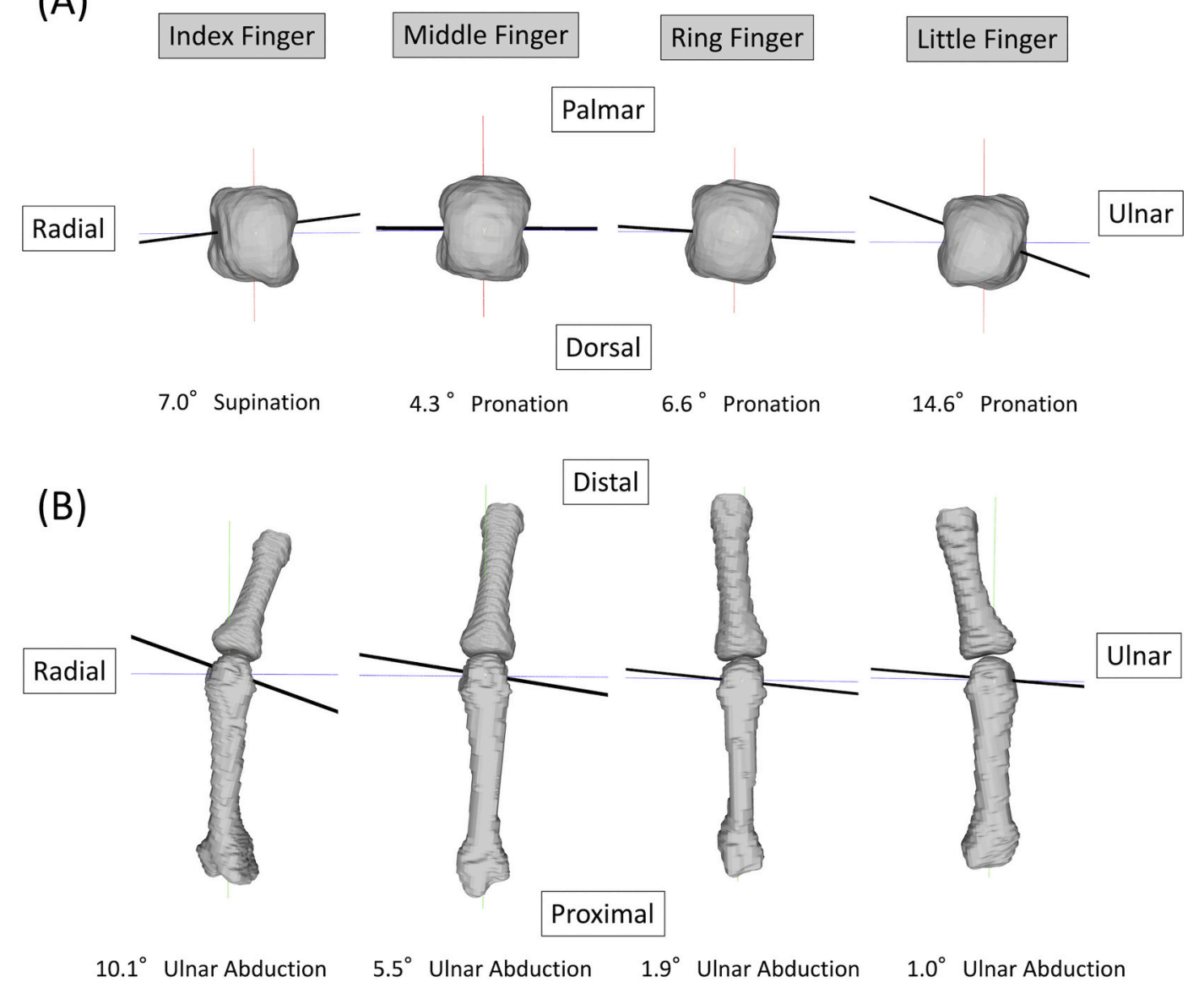

Fig. 5. Helical axes (the darkest black lines) of whole flexion were shown in the view from the axial plane (A) and coronal plane (B). The angles of the helical axes relative to the metacarpal coordinate system for 20 hands were averaged.

\section{Discussion}

We analyzed the kinematics of the MCP joints during flexion by using 4DCT and demonstrated the differences among the helical axes and rotation angles of four fingers. Our study is the first one to evaluate finger MCP joints using 4DCT. We found two interesting patterns in finger flexion. First, the index finger pronates in the early stage of flexion, and then, supinates to the end of the flexion, and the rotation range of the index finger is larger than previously reported. Second, the rotation axes of the proximal phalanx are located almost at the center of the metacarpal head and translate toward the dorsal and proximal sides during flexion. The translation is larger in the ring and little fingers compared with the index and middle fingers.

Despite these kinematic differences, most implants for MCP joint arthroplasty are designed as flexible hinge joint implants. Although the osteotomy position and size selection would partially compensate for the kinematic difference among fingers, flexibility of the silicone implant alone cannot reproduce the same function. This difference is the main reason for the short longevity of silicone implant arthroplasty for MCP joints (Trail et al., 2004). Clinically understanding the rotation axis of a joint helps design implants.

Our study reveals that the index finger supinates until $40^{\circ}$ of flexion, and then, pronates until full flexion. In contrast with the other three fingers, the function of the index finger at $40^{\circ}$ of flexion is to pinch things with the thumb. This kinematic pattern probably contributes to the pinch function in the early range of flexion and the grip function in the latter stage of flexion. When the fingers were extended, the abduction angle of the proximal phalanges relative to the metacarpals abducted ulnarly in the index finger, and radially in the ring finger and little finger to converge their fingertips. Each abduction angle approached $0^{\circ}$ accompanied by flexion, indicating that the tension of the collateral ligament increases and the tolerance of abduction decreases. Significant changes in abduction angles were observed, except for the middle finger. When the fingers were naturally extended, the abduction angle was measured as the ulnar abduction in the index finger and the radial abduction in the ring and little finger. Some factors are thought to contribute to these differences. Static factors such as asymmetric articular surfaces of the second and fifth metacarpals and dynamic factors such as contraction patterns of the flexors, extensors, and intrinsic muscles (interosseous and lumbricalis muscles) cause these kinematic differences. In particular, we believe that the anatomical morphology of the metacarpal head is most involved in the rotation. There is a discrepancy in the radius of curvature in the articular surface between the metacarpal and the base of the proximal phalange. This discrepancy is involved in the polyaxial motion of the MCP joint (Dumont et al., 2009). In the second metacarpal, the narrow dorsal articular surface is located on the ulnar side. This morphology provides a clinical anatomy that is helpful in opposing the thumb owing to the ulnar abduction and supination of the second MCP joint. In addition, the collateral ligaments are key to realize the stability of the MCP joints (Minami et al., 1985). The collateral ligaments arise from radial and ulnar tubercles located more dorsally than the center of rotation. This discrepancy causes the ligaments to loosen during extension, allowing for abduction and supination-pronation. Because the vectors of the superficial and deep digital flexor tendons are involved, in addition to the lumbrical muscle and interosseous muscle for flexion of the MCP joint, it is difficult to explain the dynamic factors unconditionally. To investigate these dynamic mechanisms, mechanical studies, such as the analysis of the 


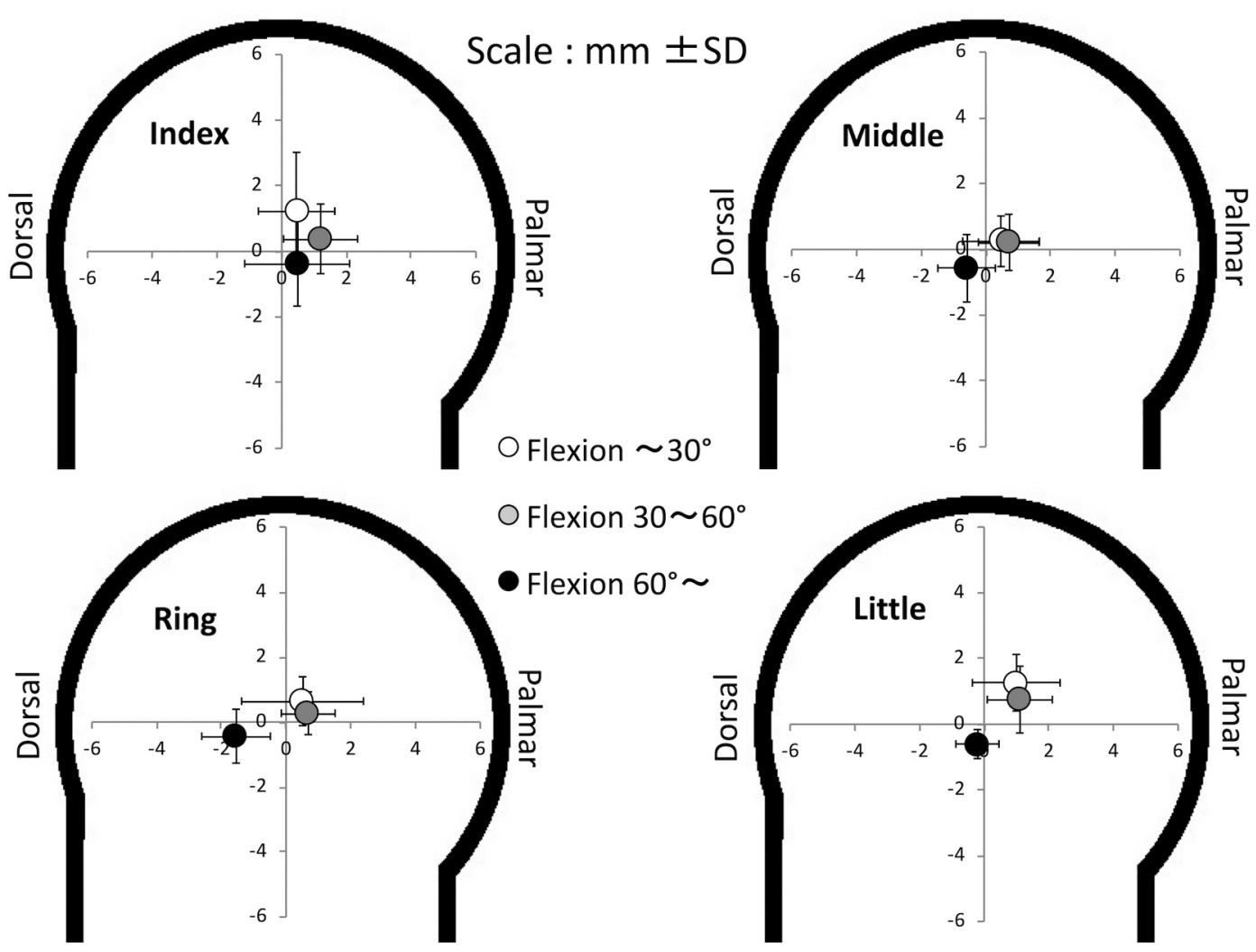

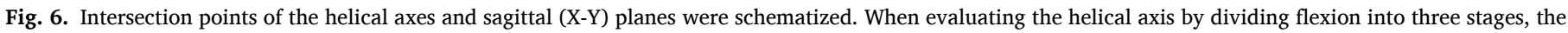
helical axis tended to move in the palmar and proximal direction at flexion $30-60^{\circ}$ and in the dorsal and proximal direction at flexion from $60^{\circ}$ on.

extension force of the MCP joint by dividing the extensor tendon and the sagittal band (Marshall et al., 2018) or the examination of the extent to which the lumbricalis muscle is involved in the flexion of the finger (Kamata et al., 2016), would be useful.

Previous studies also reported finger supination-pronation patterns using an optoelectronic system with multiple infrared markers (Buczek et al., 2011; Degeorges et al., 2005). These studies revealed similar supination patterns in the middle, ring and little fingers (Table 2). The middle, ring, and little fingers supinated during flexion, and the range of supination was larger on the ulnar side. The differences, compared with our results, are thought to result from the measurement methodology. The optoelectronic markers contain a skin motion artifact. In the analysis of skin movement related to the flexional bone motion using MR images of a hand, the skin movement of the second metacarpal was up to about $10 \mathrm{~mm}$ (Ryu et al., 2006). It is especially difficult to trace the axial rotation of the metacarpal bones that are not outwardly isolated segments. The measurement could be affected by the motions of other soft tissues, such as the skin, extensors, interosseous muscle, and fat tissue. Conversely, 4DCT can directly detect the position of the bone segments, and iterative surface registration can trace the bone segment with high accuracy because it traces the bone segment of the same individual
(Ochia et al., 2006). Therefore, this method can clarify the relationship between the kinematic patterns and anatomic landmarks of the bone segments during active motions.

Average helical axes during whole flexion were also different among the four fingers. The helical axis showed pronation in ulnar fingers, and the difference between the index and little fingers was $21^{\circ}$ in pronationsupination and $10^{\circ}$ in abduction. The MCP joint is a condylar joint with a high degree of freedom, unlike the interphalangeal joints, which are practically hinge joints. Silicon implant arthroplasty has been a widely performed technique for the treatment of MCP joints deformities in rheumatoid arthritis. However, the rate of implant fracture remains considerable (Goldfarb and Stern, 2003). The differences in the center of rotation were investigated in a study of silicone implants insertion into the MCP joint of a cadaver (Weiss et al., 2004). The use of 4DCT can be helpful in investigating silicone implant failure by examining the change of the rotation axis in in-vivo patients. There have been several reports of finite element analysis on silicon MCP implants (Hussein et al., 2011; Penrose et al., 1996). However, these studies only focused on the deformities against bending forces (flexion/extension). Further studies focusing on changes in the rotation axis will be needed to improve the design of the implants.

Table 2

Supination-pronation angles of proximal phalanges in the MCP joints during flexion were compared.

\begin{tabular}{|c|c|c|c|c|c|c|}
\hline \multirow[t]{2}{*}{ Author } & \multirow[t]{2}{*}{ Method } & \multirow[t]{2}{*}{ Participants } & \multicolumn{4}{|c|}{ Rotation angle of MCP joint (deg) } \\
\hline & & & Index & Middle & Ring & Little \\
\hline $\begin{array}{l}\text { Degeorges et al. } \\
\text { (2005) }\end{array}$ & VICON optoelectronic system & $N=6$, right hand of volunteers & $1^{\circ}$ (supination) & $5^{\circ}$ (supination) & $8^{\circ}$ (supination) & $13^{\circ}$ (supination) \\
\hline Buczek et al. (2011) & $\begin{array}{l}\text { VICON Nexus system, 6DOF } \\
\text { model }\end{array}$ & $N=20$, right hand of adults & $3.9^{\circ}$ (pronation) & $\begin{array}{l}11.8^{\circ} \\
\text { (supination) }\end{array}$ & $\begin{array}{l}15.2^{\circ} \\
\text { (supination) }\end{array}$ & $\begin{array}{l}20.9^{\circ} \\
\text { (supination) }\end{array}$ \\
\hline This study & 4DCT & $\begin{array}{l}N=10 \text {, both hands of } \\
\text { volunteers }\end{array}$ & $\begin{array}{l}6.8^{\circ} \\
\text { (supination) } \\
2.9^{\circ} \text { (pronation) }\end{array}$ & $7.2^{\circ}$ (supination) & $7.6^{\circ}$ (supination) & $9.7^{\circ}$ (supination) \\
\hline
\end{tabular}


There are several limitations exhibited by 4DCT. First, it requires radiation exposure, although the 4DCT scan of the hand does not require high radiation dosages. The radiation exposure in this study was approximately $0.06 \mathrm{mSv}$, which is comparable to the $0.05 \mathrm{mSv}$ required for chest radiography (Samara et al., 2012). Secondly, the frame rate of the 4DCT examination was relatively low ( $5 \mathrm{fps}$ ). There were almost no artifacts in $10 \mathrm{~s}$ of wrist motion; however, noises and artifacts appeared in $4 \mathrm{~s}$ of supination-pronation motion (Choi et al., 2013). In our study, a large motion artifact occurred owing to the small bone in the PIP and DIP joints. Even in the MCP joint, the registration accuracy for each frame had to be confirmed manually, and data with motion artifacts were excluded. To analyze the PIP or DIP joint, the scanning time must be extended. However, long examination times may produce joint motion that differs from daily movement.

\section{Conclusions}

In summary, quantitative analysis of the metacarpophalangeal joint during active flexion could be performed using 4DCT. The difference in the patterns of pronation-supination and radial-ulnar abduction and the helical axes for each finger was revealed. These results can be employed to better understand the causes of finger dyskinesis under pathological conditions, such as rheumatoid arthritis, fracture malunion, or implant failure.

Supplementary data to this article can be found online at https://doi. org/10.1016/j.clinbiomech.2020.105188.

\section{Funding}

This research did not receive any specific grant from funding agencies in the public, commercial, or not-for-profit sectors.

\section{Acknowledgments}

The authors do not receive funding from an external source for this research. We would like to thank Editage (www.editage.com) for the English language editing service.

\section{References}

Biswas, D., Bible, J.E., Bohan, M., Simpson, A.K., Whang, P.G., Grauer, J.N., 2009. Radiation exposure from musculoskeletal computerized tomographic scans. J. Bone Joint Surg. Am. 91, 1882-1889. https://doi.org/10.2106/JBJS.H.01199.

Buczek, F.L., Sinsel, E.W., Gloekler, D.S., Wimer, B.M., Warren, C.M., Wu, J.Z., 2011. Kinematic performance of a six degree-of-freedom hand model (6DHand) for use in occupational biomechanics. J. Biomech. 44, 1805-1809. https://doi.org/10.1016/j. jbiomech.2011.04.003.

Choi, Y.S., Lee, Y.H., Kim, S., Cho, H.W., Song, H.T., Suh, J.S., 2013. Four-dimensional real-time cine images of wrist joint kinematics using dual source CT with minimal time increment scanning. Yonsei Med. J. 54, 1026-1032. https://doi.org/10.3349/ ymj.2013.54.4.1026.

Coupier, J., Moiseev, F., Feipel, V., Rooze, M., Van Sint Jan, S., 2014. Motion representation of the long fingers: a proposal for the definitions of new anatomical frames. J. Biomech. 47, 1299-1306. https://doi.org/10.1016/j. jbiomech.2014.02.017.

Coupier, J., Hamoudi, S., Telese-Izzi, S., Feipel, V., Rooze, M., Van Sint Jan, S., 2016. A novel method for in-vivo evaluation of finger kinematics including definition of healthy motion patterns. Clin. Biomech. 31, 47-58. https://doi.org/10.1016/j. clinbiomech.2015.10.002.

Degeorges, R., Parasie, J., Mitton, D., Imbert, N., Goubier, J.N., Lavaste, F., 2005. Threedimensional rotations of human three-joint fingers: an optoelectronic measurement Preliminary results. Surg. Radiol. Anat. 27, 43-50. https://doi.org/10.1007/s00276004-0277-4.

Dumont, C., Ziehn, C., Kubein-Meesenburg, D., Fanghanel, J., Sturmer, K.M., Nagerl, H., 2009. Quantified contours of curvature in female index, middle, ring, and small metacarpophalangeal joints. J. Hand. Surg. [Am.] 34, 317-325. https://doi.org/ 10.1016/j.jhsa.2008.10.004.
Garcia-Elias, M., Alomar Serrallach, X., Monill Serra, J., 2014. Dart-throwing motion in patients with scapholunate instability: a dynamic four-dimensional computed tomography study. J. Hand Surg. Eur. 39, 346-352. https://doi.org/10.1177/ 1753193413484630.

Goldfarb, C.A., Stern, P.J., 2003. Metacarpophalangeal joint arthroplasty in rheumatoid arthritis. A long-term assessment. J. Bone Joint Surg. Am. 85, 1869-1878. https:// doi.org/10.2106/00004623-200310000-00001.

Hess, F., Furnstahl, P., Gallo, L.M., Schweizer, A., 2013. 3D analysis of the proximal interphalangeal joint kinematics during flexion. Comput. Math. Methods Med. 2013, 138063. https://doi.org/10.1155/2013/138063.

Hussein, A.I., Stranart, J.C., Meguid, S.A., Bogoch, E.R., 2011. Biomechanical validation of finite element models for two silicone metacarpophalangeal joint implants. J. Biomech. Eng. 133, 024501. https://doi.org/10.1115/1.4003311.

Kamata, Y., Nakamura, T., Tada, M., Sueda, S., Pai, D.K., Toyama, Y., 2016. How the lumbrical muscle contributes to placing the fingertip in space: a three dimensional cadaveric study to assess fingertip trajectory and metacarpophalangeal joint balancing. J. Hand Surg. Eur. Vol. 41, 386-391. https://doi.org/10.1177/ 1753193415597113.

Kataoka, T., Moritomo, H., Miyake, J., Murase, T., Yoshikawa, H., Sugamoto, K., 2011. Changes in shape and length of the collateral and accessory collateral ligaments of the metacarpophalangeal joint during flexion. J. Bone Joint Surg. Am. 93, 1318-1325. https://doi.org/10.2106/JBJS.J.00733.

Kerkhof, F.D., Brugman, E., D'Agostino, P., Dourthe, B., van Lenthe, G.H., Stockmans, F., Jonkers, I., Vereecke, E.E., 2016. Quantifying thumb opposition kinematics using dynamic computed tomography. J. Biomech. 49, 1994-1999. https://doi.org/ 10.1016/j.jbiomech.2016.05.008.

Kinzel, G.L., Hillberry, B.M., Hall Jr., A.S., Van Sickle, D.C., Harvey, W.M., 1972. Measurement of the total motion between two body segments. II. Description of application. J. Biomech. 5, 283-293. https://doi.org/10.1016/0021-9290(72) 90045-0.

Marshall, T.G., Sivakumar, B., Smith, B.J., Hile, M.S., 2018. Mechanics of metacarpophalangeal joint extension. J. Hand. Surg. [Am.] 43, 681 e681-681 e685. https://doi.org/10.1016/j.jhsa.2017.12.010.

Minami, A., An, K.N., Cooney 3rd, W.P., Linscheid, R.L., Chao, E.Y., 1985. Ligament stability of the metacarpophalangeal joint: a biomechanical study. J. Hand. Surg. [Am.] 10, 255-260. https://doi.org/10.1016/s0363-5023(85)80117-9.

Ochia, R.S., Inoue, N., Renner, S.M., Lorenz, E.P., Lim, T.H., Andersson, G.B., An, H.S., 2006. Three-dimensional in vivo measurement of lumbar spine segmental motion. Spine (Phila Pa 1976) 31, 2073-2078. https://doi.org/10.1097/01. brs.0000231435.55842.9e.

Oki, S., Kaneda, K., Yamada, Y., Yamada, M., Morishige, Y., Harato, K., Matsumura, N., Nagura, T., Jinzaki, M., 2019. Four-dimensional CT analysis using sequential 3D-3D registration. J. Vis. Exp. 153 https://doi.org/10.3791/59857.

Pagowski, S., Piekarski, K., 1977. Biomechanics of metacarpophalangeal joint. J. Biomech. 10, 205-209. https://doi.org/10.1016/0021-9290(77)90060-4.

Penrose, J.M., Williams, N.W., Hose, D.R., Trowbridge, E.A., 1996. An examination of one-piece metacarpophalangeal joint implants using finite element analysis. J. Med. Eng. Technol. 20, 145-150. https://doi.org/10.1016/s1297-3203(00)73473-1.

Ryan, T.M., Walker, A., 2010. Trabecular bone structure in the humeral and femoral heads of anthropoid primates. Anat. Rec. (Hoboken) 293, 719-729. https://doi.org/ 10.1002/ar.21139.

Ryu, J.H., Miyata, N., Kouchi, M., Mochimaru, M., Lee, K.H., 2006. Analysis of skin movement with respect to flexional bone motion using MR images of a hand. J. Biomech. 39, 844-852. https://doi.org/10.1016/j.jbiomech.2005.02.001.

Samara, E.T., Aroua, A., Bochud, F.O., Ott, B., Theiler, T., Treier, R., Trueb, P.R., Vader, J.-P., Verdun, F.R., 2012. Exposure of the Swiss population by medical X-rays. Health Phys. 102, 263-270. https://doi.org/10.1097/HP.0b013e31823513ff.

Tamai, K., Ryu, J., An, K.N., Linscheid, R.L., Cooney, W.P., Chao, E.Y., 1988. Threedimensional geometric analysis of the metacarpophalangeal joint. J. Hand. Surg. [Am.] 13, 521-529. https://doi.org/10.1016/s0363-5023(88)80088-1.

Trail, I.A., Martin, J.A., Nuttall, D., Stanley, J.K., 2004. Seventeen-year survivorship analysis of silastic metacarpophalangeal joint replacement. J. Bone Joint Surg. (Br.) 86, 1002-1006. https://doi.org/10.1302/0301-620x.86b7.15061.

Troupis, J.M., Amis, B., 2013. Four-dimensional computed tomography and trigger lunate syndrome. J. Comput. Assist. Tomogr. 37, 639-643. https://doi.org/10.1097/ RCT.0b013e31828b68ec.

Wang, K.K., Zhang, X., McCombe, D., Ackland, D.C., Ek, E.T., Tham, S.K., 2018. Quantitative analysis of in-vivo thumb carpometacarpal joint kinematics using fourdimensional computed tomography. J. Hand Surg. Eur. 43, 1088-1097. https://doi. org/10.1177/1753193418789828.

Weiss, A.P., Moore, D.C., Infantolino, C., Crisco, J.J., Akelman, E., McGovern, R.D., 2004. Metacarpophalangeal joint mechanics after 3 different silicone arthroplasties. J. Hand. Surg. [Am.] 29, 796-803. https://doi.org/10.1016/j.jhsa.2004.04.023.

Zhao, K., Breighner, R., Holmes 3rd, D., Leng, S., McCollough, C., An, K.N., 2015. A technique for quantifying wrist motion using four-dimensional computed tomography: approach and validation. J. Biomech. Eng. 137 https://doi.org/ 10.1115/1.4030405. 